\title{
Capturing the signal
}

Late-phase long-term potentiation (L-LTP) refers to LTP that persists for more than $2 \mathrm{~h}$ and results in changes in strength at excitatory synapses. When L-LTP is triggered by suprathreshold stimulation it can be 'captured' by other synapses that are subject to subthreshold stimulation. Synaptic capture has been described in apical dendrites, which arise from the apex of pyramidal cells, and Alarcon and colleagues now show that L-LTP can also be captured in basal dendrites, which emerge from the base of a pyramidal cell. Moreover, they report that this phenomenon can occur between these two types of dendritic compartment.

The initiation of L-LTP depends on the regulation of many biochemical signalling pathways that control several processes that are crucial for synaptic plasticity, including gene expression. The products of gene expression (mRNAs and proteins) are delivered throughout the cell, but it is thought that they can be captured and thereby influence increases in synaptic strength only at synapses that have been 'tagged' by previous synaptic activity.

Alarcon et al. studied L-LTP in CA1 pyramidal neurons of mice and reported that, despite differences in the mechanisms that underlie the induction and expression of LTP in basal and apical dendrites, capture of L-LTP in the basilar compartment could be induced in response to the same stimulation protocol - a single train of high-frequency $(100 \mathrm{~Hz})$ stimulation - that was used for capture in apical compartments. Capture of L-LTP in both compartments was blocked or reduced by inhibition of protein kinase A and protein synthesis, respectively, suggesting that the tagging mechanism was the same in basilar as in apical dendritic compartments.

These researchers then investigated whether capturing can take place across the two dendritic compartments. The stimulation protocol used to induce within-compartment capture was not sufficient to drive capture between the two compartments in either direction. Varying the intensity of the priming stimuli, the time interval between stimuli delivered to each dendritic compartment, and the distance between the nucleus and the capture site did not allow cross-compartment capture. However, this could be triggered following stronger activation, such as two trains of tetanic stimulation.

Alarcon and colleagues propose that, although the tagging signal is the same in basilar and apical compartments, the synaptic tag is compartment restricted after only one train of stimulation; heightened stimulation is thought to trigger synaptic tagging over a wider area, allowing cross-compartment capture to take place. This, they say, would allow hippocampal neurons to process dendritic signals differently depending on the level of activation. However, further work will be necessary to confirm this model and unravel the principles that govern activity-mediated synaptic capture between different dendritic compartments.

Alison Rowan

ORIGINAL RESEARCH PAPER Alarcon, J. M., Barco, A. \& Kandel, E. R. Capture of the late phase of long-term potentiation within and across the apical and basilar dendritic compartments of CA1 pyramidal neurons: synaptic tagging is compartment restricted. J. Neurosci. 26, 256-264 (2006)

FURTHER READING Frey, U. \& Morris, R. G. Synaptic tagging and long-term potentiation. Nature 385, 533-536 (1997) | Martin, K. C. \& Kosik, K. S. Synaptic tagging - who's it? Nature Rev. Neurosci. 3, 813-820 (2002)| Adams, J. P. \& Dudek, S. M. Late-phase long-term potentiation: getting to the nucleus. Nature Rev. Neurosci. 6, 737-743 (2005) 